\title{
Global Longitudinal Strain: Is It Time to Change the Preoperative Cardiac Assessment of Oncology Patients?
}

\author{
Islam Mohammad Shehata · Tiffany D. Odell · Amir Elhassan • \\ Ivan Urits · Omar Viswanath · Alan D. Kaye
}

Received: August 24, 2020 / Accepted: November 10, 2020 / Published online: November 28, 2020

(C) The Author(s) 2020

\section{ABSTRACT}

The introduction of new anticancer treatment modalities has improved survival rates, transforming cancer into a chronic disease in many instances. One of the most devastating complications of cancer treatment is cancer therapyrelated cardiac dysfunction. Adequate preoperative assessment of any significant cancer therapy-related cardiac impairment is critical, and may be missed with conventional measures. The assessment of global longitudinal strain by speckle-tracking echocardiography is more sensitive for the early detection of cardiac contractility before a decline in ejection fraction can be discovered. Global longitudinal strain

I. M. Shehata

Department of Anesthesiology, Faculty of Medicine, Ain Shams University, Cairo, Egypt

T. D. Odell

Department of Neurosurgery, Desert Regional

Medical Center, Palm Springs, CA, USA

A. Elhassan

Cardiothoracic Anesthesia, Desert Regional Medical Center, Palm Springs, CA, USA

I. Urits · O. Viswanath · A. D. Kaye $(\square)$

Department of Anesthesiology, Louisiana State

University Health Shreveport, Shreveport, LA, USA

e-mail: akaye@lsuhsc.edu can also predict postoperative cardiac dysfunction, which makes it a good alternative for preoperative cardiac assessment in the oncology population when cancer therapies have been administered that can alter normal performance.

Keywords: Cancer therapy-related cardiac dysfunction; Cardiac dysfunction; Global longitudinal strain; Heart failure; Left ventricular ejection fraction; Oncology; Preoperative cardiac assessment; Speckletracking echocardiography

\footnotetext{
I. Urits

Department of Anesthesia, Critical Care, and Pain Medicine, Harvard Medical School, Beth Israel Deaconess Medical Center, Boston, MA, USA

O. Viswanath

Valley Anesthesiology and Pain Consultants, Envision Physician Services, Phoenix, AZ, USA

O. Viswanath

Department of Anesthesiology, Creighton

University School of Medicine, Omaha, NE, USA

O. Viswanath

University of Arizona College of Medicine-Phoenix, Phoenix, AZ, USA
} 


\section{Key Summary Points}

Cancer has become a chronic disease due to the introduction of new anticancer strategies.

One of the most devastating side effects of cancer treatment is the cancer therapyrelated cardiac dysfunction. which should be considered during the preoperative workup of cancer patients.

Traditional 2D echocardiography is limited in its ability to accurately detect left ventricular ejection fraction (LVEF).

Speckle-tracking echocardiography is an alternative imaging modality which uses global longitudinal strain and has been shown to improve reliability in the measurement of LVEF.

The measurement of left ventricular contractile function by global longitudinal strain might be a more sensitive predictor of cardiac dysfunction, because myocardial deformation precedes LVEF changes in patients undergoing anticancer therapy.

Measurement of global longitudinal strain by speckle-tracking echocardiography should be used routinely in the preoperative workup of patients with a history of cancer in order to detect cancer therapy-related cardiac dysfunction.

\section{DIGITAL FEATURES}

This article is published with digital features, including a summary slide, to facilitate understanding of the article. To view digital features for this article go to https://doi.org/10.6084/ m9.figshare.13208216.

\section{COMMENTARY}

Cancer is defined as uncontrolled growth and spread of abnormal cells, which can result in death if not controlled [1]. The annual value of lost work productivity due to early mortality from cancer in the United States was approximately $\$ 115.8$ billion in 2000 , with the cost projected to increase to $\$ 147.6$ billion by 2020 . A $1 \%$ annual reduction in lung, colorectal, breast, leukemia, pancreatic, and brain cancer mortality would translate to an astounding $\$ 814$ million reduction in lost work productivity cost per year [2].

The treatment modalities for cancer traditionally comprise a multidisciplinary approach involving a combination of chemotherapy, radiotherapy, and surgery to prolong life expectancy and decrease the global burden of disability [3]. However, the introduction of new anticancer strategies that have improved survival rates has transformed cancer into a chronic disease. As a result, there is a growing population of patients with a history of cancer who have required various surgical interventions. In 2014, there were an estimated 14.5 million American cancer survivors, a number which is expected to reach 18 million by 2020 [4]. Thus, the need for surgery in cancer survivors has called attention to the side effects of cancer treatment, which should be taken into consideration during risk assessment and stratification in the preoperative period.

Although there have been many advances in cancer treatment, one of the most devastating side effects of cancer treatment is cancer therapy-related cardiac dysfunction (CTRCD). The generally accepted definition of CTRCD is a reduction from baseline left ventricular ejection fraction (LVEF) of greater than $5 \%$ to a value $<55 \%$, in addition to signs or symptoms of heart failure (HF), or a reduction in LVEF > $10 \%$ to an $\mathrm{LVEF}<55 \%$, without signs or symptoms of HF. Because of the causative nature of the anticancer treatments, the development of CTRCD necessitates interruption of the 
ongoing anticancer therapy [5]. The American College of Cardiology/American Heart Association (ACC/AHA) and Heart Failure Society of America classify cancer survivors receiving cardiotoxic chemotherapy as being at least stage A HF (at high risk for HF but without structural heart disease or symptoms of HF) [6]. There is also a causal relationship between certain side effects and specific chemotherapy agents [7]. Many risk factors promote the development of these side effects as well, including pre-existing cardiac diseases, diabetes mellitus, hypertension, and hyperlipidemia [8].

The European Society of Cardiology guidelines divide CTRCD into nine major categories [9]. The cardiovascular effects include heart failure, arrhythmias, vascular complications such as hypotension or hypertension, coronary ischemia, valvular heart disease, and pericardial disease. Overall, CTRCD has been linked to a 3.5-fold increased mortality risk compared with idiopathic cardiomyopathy [10]. This has significant implications for the prognosis and survival of cancer patients and should be considered in preoperative patient assessment. Additionally, early identification of CTRCD may aid in mitigating morbidity that may be encountered during surgery, such as intraoperative hypotension.

CTRCD can increase the risk of intraoperative hypotension, especially the post-induction type $[11,12]$. A consistent relationship has been found between intraoperative hypotension and postoperative acute renal impairment and myocardial injury in non-cardiac surgery [13]. Therefore, the anesthesiologist should be vigilant for CTRCD, which may necessitate a change in the anesthesia plan to avoid intraoperative hypotension and its deleterious consequences. The preoperative detection of CTRCD may influence the choice of induction agents, and the need to consider more hemodynamically stable agents and a reduction in the overall doses. Furthermore, identifying this important risk factor may guide the use of invasive monitoring and transesophageal echocardiography, especially in extensive surgery with higher risk of bleeding.

Several molecular theories have been proposed to explain CTRCD [14]. The most compelling pathophysiological mechanism is myocardial cell death due to either apoptosis or loss of myofibrils and vacuolization of myocytes. Various recently reported data implicate altered topoisomerase activity as a key mediator of this pathology [15].

Indirect effects include impairment of endogenous cardioprotective mechanisms such as activation of antioxidant activity, growth factors, nitric oxide, gp130, and the neuregulin/ ErbB signaling system [16].

Traditionally, left ventricular ejection fraction (LVEF) has been assessed by 2D echocardiography. It is the most widely used technique because of international availability and its low cost in comparison to other imaging modalities. Lack of radiation exposure and ability to assess other pathology such as valvular or pericardial disease are additional benefits. However, 2D echocardiography may be inadequate for proper assessment in this population.

LVEF assessment by 2D echocardiography has demonstrated limited reliability, with the ability to detect only those LVEF differences over time which are greater than $10 \%$. The temporal variability between measurements is attributed to the suboptimal biological (day-today) reproducibility of LVEF, which is about $10 \%$ in both the general and oncologic populations [17]. It is critical to understand that such a drop in LVEF is a late event in CTRCD, and thus is not detectable until significant cardiac damage has already occurred. Therefore, more sensitive and specific diagnostic tools are needed for earlier diagnosis of CTRCD.

Cardiac investigations for timely management of cardiac toxicity include invasive and noninvasive techniques. Noninvasive imaging includes standard and advanced tools. Advanced imaging includes (speckle-tracking and three-dimensional) echocardiography, cardiac magnetic resonance imaging (CMRI), cardiac computed tomography (CCT) and cardiac radionuclide imaging, and single-photon emission cardiac tomography (SPECT) [18].

An alternative imaging modality, speckletracking echocardiography (STE), has been shown to improve reliability in the measurement of LVEF. The assessment of global longitudinal strain (GLS) by STE has demonstrated 


\section{Preoperative Cardiac Assessment of Oncology Patients}

- Detailed Clinical History (exercise tolerance/chemotherapy drugs)

- Focused cardiovascular examination

\section{LVEF}

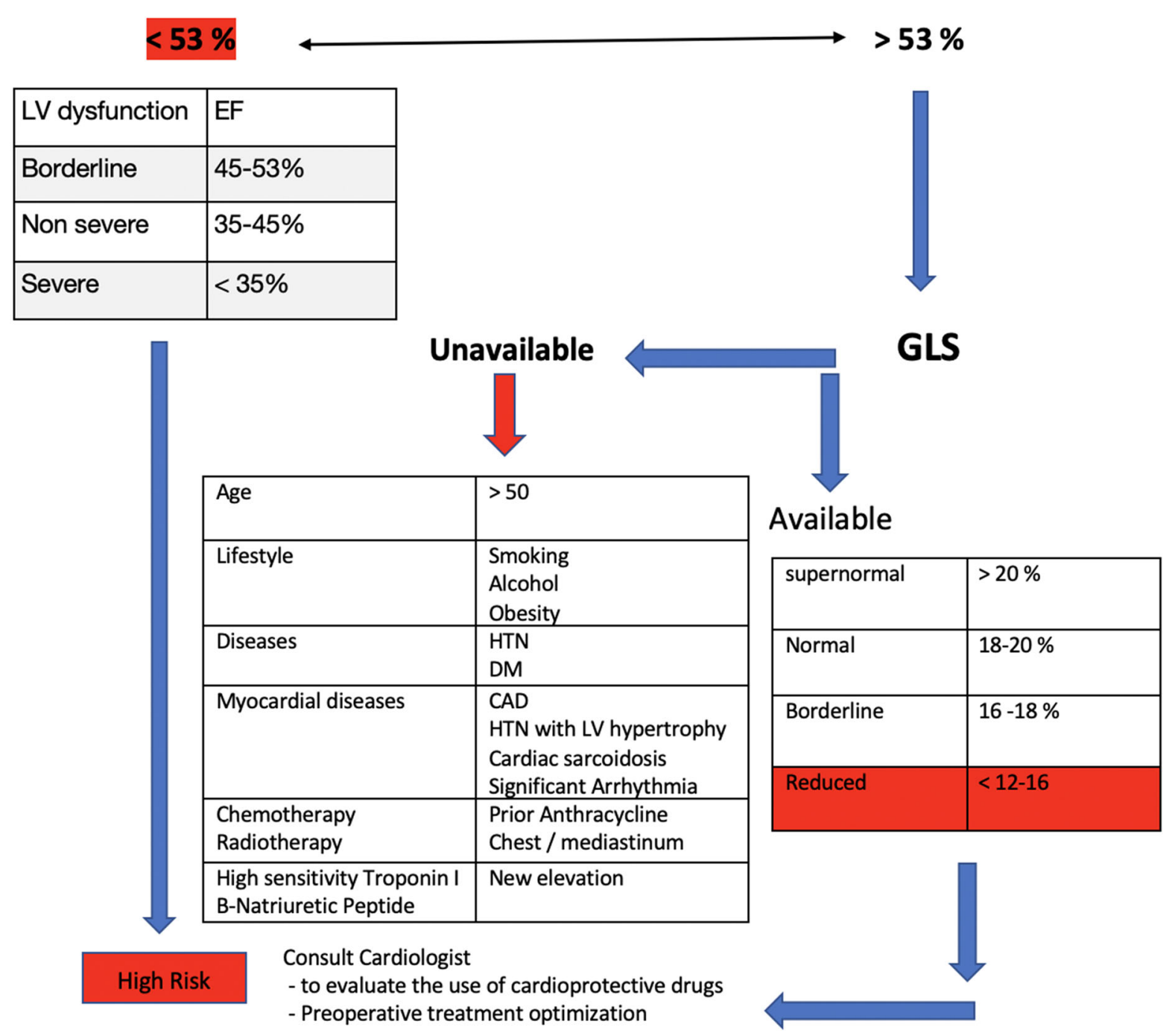

Fig. 1 Preoperative cardiac assessment algorithm for oncology patients. $L V E F$ left ventricular ejection fraction, $G L S$ global longitudinal strain, $H T N$ hypertension, $D M$

biological reproducibility of $6 \%$ [19]. Therefore, measurement of left ventricular contractile function by GLS might be more sensitive, given diabetes mellitus, CAD coronary artery disease. ${ }^{*}$ Red boxes, arrows refer to high-risk patients

that myocardial deformation precedes LVEF changes in patients undergoing anticancer therapy. Furthermore, a $10 \%$ to $15 \%$ early 
reduction in GLS, as measured by STE during anticancer therapy, appears to be a useful independent predictor of cardiac toxicity, defined as a drop in LVEF or heart failure [20].

Therefore, GLS can be used to initiate timely cardiac protective measures in the treatment of the oncology population to avoid progression from subclinical CTRCD to overt heart failure [21]. With regard to perioperative management, a prospective study including 88 patients showed that preoperative GLS may be effective in predicting postoperative left ventricular dysfunction [22]. Another study involving 250 patients demonstrated that right ventricular (RV) GLS is a sensitive marker of RV dysfunction and correlates with postoperative mortality [23]. The SUCCOUR trial was a randomized clinical trial which demonstrated that GLS was more precise than 2D echocardiography specifically in the oncology population [24].

In order to diagnose underlying CTRCD and to identify and mitigate risk in the perioperative period, the authors propose a preoperative algorithm for the assessment of oncology patients which addresses this issue. Considering the current literature, surveillance of cardiac dysfunction in this high-risk population would be prudent. Traditional preoperative assessment may fail to identify CTRCD. Below is a proposed preoperative cardiac assessment algorithm which integrates clinical history, imaging, and biomarkers to provide a comprehensive assessment in this population. This would ensure proper preoperative assessment and may serve to mitigate risk, morbidity, and mortality associated with the oncology population.

\section{PREOPERATIVE CARDIAC ASSESSMENT ALGORITHM (FIG. 1)}

The cardiac assessment should include detailed clinical history to identify the risk factors for CTRCD. Risk factors include prior chemotherapy treatment, current cardiac disorders, and lifestyle habits including smoking, alcohol use, and sedentary lifestyle [25]. The main disadvantages of GLS are the technical considerations and dependence on the quality of the 2D images, which necessitates further training and education [26]. Moreover, because of the greater availability of LVEF over GLS, the former remains a cornerstone of preoperative assessment and therapeutic decision-making. However, the value of GLS is more significant in the detection of asymptomatic left ventricular dysfunction, especially with preserved LVEF. The GLS can reclassify apparently normal LVEF and detect patients at high risk for all-cause mortality [27]. If GLS is not feasible, the perioperative physicians should integrate the history of risk factors, focused cardiac examination, and the cardiac biomarkers to identify high-risk patients. New elevation of cardiac troponins and natriuretic peptides can be used for the diagnosis of myocardial injury [21]. In those high-risk patients, the cardiologist should be involved to initiate cardioprotective drugs or optimize preoperative treatment.

Therefore, the assessment of cardiac function by GLS should be considered as a routine preoperative cardiac assessment in the oncology population.

\section{ACKNOWLEDGEMENTS}

Funding. No funding or sponsorship was received for this study or publication of this article.

Authorship. All named authors meet the International Committee of Medical Journal Editors (ICMJE) criteria for authorship for this article, take responsibility for the integrity of the work as a whole, and have given their approval for this version to be published.

Disclosures. Islam Mohammad Shehata, MD; Tiffany D. Odell, DO; Amir Elhassan, MD; Ivan Urits, MD; Omar Viswanath, MD; and Alan D. Kaye, MD, PhD have nothing to disclose.

Compliance with Ethics Guidelines. This article is based on previously conducted studies and does not contain any studies with human participants or animals performed by any of the authors. 
Data Availability. Data sharing is not applicable to this article as no datasets were generated or analyzed during the current study.

Open Access. This article is licensed under a Creative Commons Attribution-NonCommercial 4.0 International License, which permits any non-commercial use, sharing, adaptation, distribution and reproduction in any medium or format, as long as you give appropriate credit to the original author(s) and the source, provide a link to the Creative Commons licence, and indicate if changes were made. The images or other third party material in this article are included in the article's Creative Commons licence, unless indicated otherwise in a credit line to the material. If material is not included in the article's Creative Commons licence and your intended use is not permitted by statutory regulation or exceeds the permitted use, you will need to obtain permission directly from the copyright holder. To view a copy of this licence, visit http:// creativecommons.org/licenses/by-nc/4.0/.

\section{REFERENCES}

1. Are C, Caniglia A, Mohammed M, et al. Global variations in the level of cancer-related research activity and correlation to cancer-specific mortality: proposal for a global curriculum. Ann Surg Oncol. 2018;25(3):594-603.

2. Bradley CJ, Yabroff KR, Dahman B, Feuer EJ, Mariotto A, Brown ML. Productivity costs of cancer mortality in the United States: 2000-2020. J Natl Cancer Inst. 2008;100(24):1763-70.

3. Geiger S, Lange V, Suhl P, Heinemann V, Stemmler HJ. Anticancer therapy induced cardiotoxicity: review of the literature. Anticancer Drugs. 2010;21(6):578-90.

4. DeSantis CE, Lin CC, Mariotto AB, et al. Cancer treatment and survivorship statistics, 2014. CA Cancer J Clin. 2014;64(4):252-71.

5. Seidman A, Hudis C, Pierri MK, et al. Cardiac dysfunction in the trastuzumab clinical trials experience. J Clin Oncol. 2002;20(5):1215-21.

6. Hunt SA, Abraham WT, Chin MH, et al. 2009 Focused update incorporated into the ACC/AHA
2005 guidelines for the diagnosis and management of heart failure in adults: a report of the American College of Cardiology Foundation/American Heart Association Task Force on Practice Guidelines developed in collaboration with the International Society for Heart and Lung Transplantation. J Am Coll Cardiol. 2009;53(15):e1-90.

7. Oprea AD, Russell RR, Russell KS, Abu-Khalaf M. Chemotherapy agents with known cardiovascular side effects and their anesthetic implications. J Cardiothorac Vasc Anesth. 2017;31(6):2206-26.

8. Floyd JD, Nguyen DT, Lobins RL, Bashir Q, Doll DC, Perry MC. Cardiotoxicity of cancer therapy. J Clin Oncol. 2005;23(30):7685-96.

9. Zamorano JL, Lancellotti P, Rodriguez Muñoz D, et al. 2016 ESC Position Paper on cancer treatments and cardiovascular toxicity developed under the auspices of the ESC Committee for Practice Guidelines: the Task Force for cancer treatments and cardiovascular toxicity of the European Society of Cardiology (ESC). Eur Heart J. 2016;37(36): 2768-801.

10. Felker GM, Thompson RE, Hare JM, et al. Underlying causes and long-term survival in patients with initially unexplained cardiomyopathy. N Engl J Med. 2000;342(15):1077-84.

11. Yeh E, Bickford CL. Cardiovascular complications of cancer therapy: incidence, pathogenesis, diagnosis, and management. J Am Coll Cardiol. 2009;53(24):2231-47.

12. Südfeld S, Brechnitz S, Wagner JY, Reese PC, Pinnschmidt HO, Reuter DA, Saugel B. Post-induction hypotension and early intraoperative hypotension associated with general anaesthesia. $\mathrm{Br}$ J Anaesthesia. 2017;119(1):57-64.

13. Walsh M, Devereaux PJ, Garg AX, Kurz A, Turan A, Rodseth RN, Cywinski J, Thabane L, Sessler DI. Relationship between intraoperative mean arterial pressure and clinical outcomes after noncardiac surgery: toward an empirical definition of hypotension. Anesthesiology. 2013;119(3):507-15.

14. Han X, Zhou Y, Liu W. Precision cardio-oncology: understanding the cardiotoxicity of cancer therapy. NPJ Precis Oncol. 2017;1(1):1-1.

15. Zhang S, Liu X, Bawa-Khalfe T, et al. Identification of the molecular basis of doxorubicin-induced cardiotoxicity. Nat Med. 2012;18(11):1639.

16. Zuppinger C, Timolati F, Suter TM. Pathophysiology and diagnosis of cancer drug induced cardiomyopathy. Cardiovasc Toxicol. 2007;7(2):61-6. 
17. Thavendiranathan P, Grant AD, Negishi T, Plana JC, Popović ZB, Marwick TH. Reproducibility of echocardiographic techniques for sequential assessment of left ventricular ejection fraction and volumes: application to patients undergoing cancer chemotherapy. J Am Coll Cardiol. 2013;61(1): 77-84.

18. Galderisi M, Lancellotti P. What is the best imaging tool in cardio-oncology?.

19. Farsalinos KE, Daraban AM, Ünlü S, Thomas JD, Badano LP, Voigt JU. Head-to-head comparison of global longitudinal strain measurements among nine different vendors: the EACVI/ASE Inter-Vendor Comparison Study. J Am Soc Echocardiogr. 2015;28(10):1171-81.

20. Thavendiranathan P, Poulin F, Lim KD, Plana JC, Woo A, Marwick TH. Use of myocardial strain imaging by echocardiography for the early detection of cardiotoxicity in patients during and after cancer chemotherapy: a systematic review. J Am Coll Cardiol. 2014;63(25 Part A):2751-68.

21. Plana JC, Galderisi M, Barac A, et al. Expert consensus for multimodality imaging evaluation of adult patients during and after cancer therapy: a report from the American Society of Echocardiography and the European Association of Cardiovascular Imaging. Eur Heart J Cardiovasc Imaging. 2014;15(10):1063-93.

22. Mascle S, Schnell F, Thebault C, et al. Predictive value of global longitudinal strain in a surgical population of organic mitral regurgitation. J Am Soc Echocardiogr. 2012;25(7):766-72.

23. Ternacle J, Berry $M$, Cognet $T$, et al. Prognostic value of right ventricular two-dimensional global strain in patients referred for cardiac surgery. J Am Soc Echocardiogr. 2013;26(7):721-6.

24. Negishi T, Thavendiranathan P, Penicka M, Deblois J, Aakhus S, Miyazaki S, Shirazi M, Galderisi M, Vinereanu D, Cho J-Y, Hristova K, Popescu B, Marwick T, Negishi K. Precision and stability of parameters for assessment of left ventricular systolic function in clinical trails: lessons from the SUCCOUR trial. J Am Coll Cardiol. 2019;73(9):1514.

25. Lopez-Fernandez T, García AM, Beltrán AS, Luis ÁM, Sanz RG, Ramos PM, del Castillo SV, de Sa Areses EL, Barreiro-Perez M, Baydes RH, de Isla LP. Cardio-onco-hematology in clinical practice. Position paper and recommendations. Rev Española Cardiol (English Edition). 2017;70(6):474-86.

26. Negishi T, Negishi K, Thavendiranathan P, Cho GY, Popescu BA, Vinereanu D, Kurosawa K, Penicka M, Marwick TH, Aakhus S, Bansal M. Effect of experience and training on the concordance and precision of strain measurements. JACC Cardiovasc Imaging. 2017;10(5):518-22.

27. Potter E, Marwick TH. Assessment of left ventricular function by echocardiography: the case for routinely adding global longitudinal strain to ejection fraction. JACC Cardiovasc Imaging. 2018;11(2 Part 1):260-74. 